\title{
Existence of Solution and Approximate Controllability for Neutral Differential Equation with State Dependent Delay
}

\author{
Sanjukta Das, Dwijendra N. Pandey, and N. Sukavanam \\ Department of Mathematics, IIT Roorkee, Roorkee, Uttarakhand 247667, India \\ Correspondence should be addressed to Dwijendra N. Pandey; dwij.iitk@gmail.com
}

Received 12 June 2014; Revised 10 September 2014; Accepted 15 September 2014; Published 2 October 2014

Academic Editor: Athanasios N. Yannacopoulos

Copyright ( 2014 Sanjukta Das et al. This is an open access article distributed under the Creative Commons Attribution License, which permits unrestricted use, distribution, and reproduction in any medium, provided the original work is properly cited.

\begin{abstract}
This paper is divided in two parts. In the first part we study a second order neutral partial differential equation with state dependent delay and noninstantaneous impulses. The conditions for existence and uniqueness of the mild solution are investigated via Hausdorff measure of noncompactness and Darbo Sadovskii fixed point theorem. Thus we remove the need to assume the compactness assumption on the associated family of operators. The conditions for approximate controllability are investigated for the neutral second order system with respect to the approximate controllability of the corresponding linear system in a Hilbert space. A simple range condition is used to prove approximate controllability. Thereby, we remove the need to assume the invertibility of a controllability operator used by authors in (Balachandran and Park, 2003), which fails to exist in infinite dimensional spaces if the associated semigroup is compact. Our approach also removes the need to check the invertibility of the controllability Gramian operator and associated limit condition used by the authors in (Dauer and Mahmudov, 2002), which are practically difficult to verify and apply. Examples are provided to illustrate the presented theory.
\end{abstract}

\section{Introduction}

Neutral differential equations appear as mathematical models in electrical networks involving lossless transmission, mechanics, electrical engineering, medicine, biology, ecology, and so forth. Neutral differential equations are functional differential equations in which the highest order derivative of the unknown function appears both with and without derivatives. Second order neutral differential equations model variational problems in calculus of variation and appear in the study of vibrating masses are attached to an electric bar.

Impulsive differential equations are known for their utility in simulating processes and phenomena subject to short term perturbations during their evolution. Discrete perturbations are negligible to the total duration of the process which have been studied in [1-6].

However noninstantaneous impulses are recently studied by Ahmad [7]. Stimulated by their numerous applications in mechanics, electrical engineering, medicine, ecology, and so forth, noninstantaneous impulsive differential equations are recently investigated.

Recently, much attention is paid to partial functional differential equation with state dependent delay. For details see [7-12]. As a matter of fact, in these papers their authors assume severe conditions on the operator family generated by $A$, which imply that the underlying space $X$ has finite dimension. Thus the equations treated in these works are really ordinary and not partial equations. The literature related to state dependent delay mostly deals with functional differential equations in which the state belongs to a finite dimensional space. As a consequence, the study of partial functional differential equations with state dependent delay is neglected. This is one of the motivations of our paper.

The papers $[13,14]$ study existence of differential equation via measure of noncompactness. Measure of noncompactness significantly removes the need to assume Lipschitz continuity of nonlinear functions and operators.

In recent years, controllability of infinite dimensional systems has been extensively studied for various applications. In 
the papers $[15,16]$ the authors discuss the exact controllability results by assuming that the semigroup associated with the linear part is compact. However, if the operator $B$ is compact or $C_{0}$-semigroup $T(t)$ is compact then the controllability operator is also compact. Hence the inverse of it does not exist if the state space $X$ is infinite dimensional [17].

Another available method in the literature involves the invertibility of operator $\left(\alpha I+\Gamma_{0}^{T}\right)$, where $\Gamma_{0}^{T}$ is the controllability Gramian and a limit condition which is difficult to check and apply in practical real world problems. See for details [18]. Also it is practically difficult to verify their condition directly. This is one of the motivations of our paper.

However our work is a continuation of coauthor Sukavanam's novel approach in article [19]. We extend our work [20-22] in this paper.

Controllability results are available in overwhelming majority for abstract differential delay systems (see [1, 3-6, 9$12,14-17,19-34])$, rather than for neutral differential with state dependent delay.

The organization of the paper is as follows. In Section 3 we study the existence and uniqueness of mild solution of the second order equation modelled in the form

$$
\begin{array}{r}
\frac{d}{d t}\left(x^{\prime}(t)+g\left(t, x_{t}\right)\right)=A x(t)+f\left(t, x_{\rho\left(t, x_{t}\right)}\right), \\
t \in\left(s_{i}, t_{i+1}\right], \quad i=0, \ldots, n, \\
x_{0}=\phi \in \mathfrak{B}, \quad x^{\prime}(0)=z \in X, \\
x(t)=J_{i}^{1}\left(t, x_{t}\right), \quad t \in\left(t_{i}, s_{i}\right], i=1,2, \ldots, n, \\
x^{\prime}(t)=J_{i}^{2}\left(t, x_{t}\right), \quad t \in\left(t_{i}, s_{i}\right], \quad i=1,2, \ldots, n,
\end{array}
$$

where $A$ is the infinitesimal generator of a strongly continuous cosine family $\{C(t): t \in \mathbb{R}\}$ of bounded linear operators on a Banach space $X$. The history valued function $x_{t}:(-\infty, 0] \rightarrow X, x_{t}(\theta)=x(t+\theta)$ belongs to some abstract phase space $\mathfrak{B}$ defined axiomatically; $g, f, J_{i}^{1}, J_{i}^{2}, i=1, \ldots, n$ are appropriate functions. $0=t_{0}=s_{0}<t_{1} \leq s_{1} \leq t_{2},<\cdots,<$ $t_{n} \leq s_{n} \leq t_{n+1}=a$ are prefixed numbers. In Section 5 we study the approximate controllability of

$$
\begin{gathered}
\frac{d}{d t}\left(x^{\prime}(t)+g\left(t, x_{t}\right)\right)=A x(t)+f\left(t, x_{\rho\left(t, x_{t}\right)}\right)+B u(t), \\
t \in J=[0, a], \\
x_{0}=\phi \in \mathfrak{B}, \quad x^{\prime}(0)=w \in X,
\end{gathered}
$$

where $A$ is the infinitesimal generator of a strongly continuous cosine family $\{C(t): t \in \mathbb{R}\}$ of bounded linear operators on a Hilbert space $X$. The history valued function $x_{t}:(-\infty, 0] \rightarrow X, x_{t}(\theta)=x(t+\theta)$ belongs to some abstract phase space $\mathfrak{B}$ defined axiomatically; $g, f$ are appropriate functions. $B$ is a bounded linear operator on a Hilbert space $U$.

\section{Preliminaries}

In this section some definitions, notations, and lemmas that are used throughout this paper are stated. The family $\{C(t)$ : $t \in \mathbb{R}\}$ of operators in $B(X)$ is a strongly continuous cosine family if the following are satisfied:

(a) $C(0)=I(I$ is the identity operator in $X)$;

(b) $C(t+s)+C(t-s)=2 C(t) C(s)$ for all $t, s \in \mathbb{R}$;

(c) the map $t \rightarrow C(t) x$ is strongly continuous for each $x \in X$.

$\{S(t): t \in \mathbb{R}\}$ is the strongly continuous sine family associated to the strongly continuous cosine family $\{C(t): t \in \mathbb{R}\}$. It is defined as $S(t) x=\int_{0}^{t} C(s) x d s, x \in X, t \in \mathbb{R}$.

The operator $A$ is the infinitesimal generator of a strongly continuous cosine function of bounded linear operators $(C(t))_{t \in \mathbb{R}}$ and $S(t)$ is the associated sine function. Let $N, \widetilde{N}$ be certain constants such that $\|C(t)\| \leq N$ and $\|S(t)\| \leq \widetilde{N}$ for every $t \in J=[0, a]$. For more details see book by Fattorini [28] and articles [35-37]. In this work we use the axiomatic definition of phase space $\mathfrak{B}$, introduced by Hale and Kato [30].

Definition 1 (see [30]). Let $\mathfrak{B}$ be a linear space of functions mapping $(-\infty, 0]$ into $X$ endowed with seminorm $\|\cdot\|_{\mathfrak{B}}$ and satisfy the following conditions:

(A) If $x:(-\infty, \sigma+b] \rightarrow X, b>0$, such that $x_{\theta} \in \mathfrak{B}$ and $\left.x\right|_{[\sigma, \sigma+b]} \in C([\sigma, \sigma+b]: X)$, then for every $t \in[\sigma, \sigma+b)$ the following conditions:

(i) $x_{t}$ is in $\mathfrak{B}$,

(ii) $\|x(t)\| \leq H\left\|x_{t}\right\|_{\mathfrak{B}}$,

(iii) $\left\|x_{t}\right\|_{\mathfrak{B}} \leq K(t-\sigma) \sup \{\|x(s)\|: \sigma \leq s \leq t\}+M(t+$ $\sigma)\left\|x_{\sigma}\right\|_{\mathfrak{B}}$

where $H>0$ is a constant $K, M:[0, \infty) \rightarrow$ $[1, \infty), K$ is continuous, $M$ is locally bounded and $H, K, M$ are independent of $x(\cdot)$.

(B) The space $\mathfrak{B}$ is complete.

Definition 2 (see [31]). Hausdorff's measure of noncompactness $\chi_{Y}$ for a bounded set $B$ in any Banach space $Y$ is defined by $\chi_{Y}(B)=\inf \{r>0, B$ can be covered by finite number of balls with radii $r$.

Lemma 3 (see [31]). Let $Y$ be a Banach space and $B, C \subset Y$ be bounded, then the following properties hold:

(1) $B$ is precompact if and only if $\chi_{Y}(B)=0$;

(2) $\chi_{Y}(B)=\chi_{Y}(\bar{B})=\chi_{Y}(\operatorname{conv} B)$, where $\bar{B}$ and $\operatorname{conv} B$ are closure and convex hull of $B$, respectively;

(3) $\chi_{Y}(B) \leq \chi_{Y}(C)$ when $B \subset C$;

(4) $\chi_{Y}(B+C) \leq \chi_{Y}(B)+\chi_{Y}(C)$ where $B+C=\{x+y ; x \in$ $B, y \in C\}$;

(5) $\chi_{Y}(B \cup C)=\max \left\{\chi_{Y}(B), \chi_{Y}(C)\right\}$; 
(6) $\chi_{Y}(\lambda B)=\|\lambda\| \chi_{Y}(B)$ for any $\lambda \in \mathbb{R}$;

(7) if the map $Q: D(Q) \subset Y \rightarrow Z$ is Lipschitz continuous with constant $k$ then $\chi_{Z}(Q B) \leq k \chi_{Y}(B)$ for any bounded subset $B \subset D(Q)$, where $Z$ is a Banach space;

(8) if $\left\{W_{n}\right\}_{n=1}^{+\infty}$ is a decreasing sequence of bounded closed nonempty subset of $Y$ and $\lim _{n \rightarrow \infty} \chi_{Y}\left(W_{n}\right)=0$, then $\bigcap_{n=1}^{+\infty} W_{n}$ is nonempty and compact in $Y$.

Definition 4 (see [31]). The map $Q: W \subset Y \rightarrow Y$ is said to be a $\chi$-contraction if there exists a positive constant $k<1$ such that $\chi_{Y} Q(C) \leq k \chi_{Y}(C)$ for any bounded close subset $C \subset W$ where $Y$ is a Banach space.

Lemma 5 (Darbo-Sadovskii [31]). If $W \subset Y$ is closed and convex and $0 \in W$, the continuous map $Q: W \rightarrow W$ is $\chi$-contraction, then the map $Q$ has at least one fixed point.

$\operatorname{PC}([0, a], X)$ is the space formed by normalized piecewise continuous function from $[0, b]$ into $X$. In particular it is the space PC formed by all functions $u:[0, b] \rightarrow X$ such that $u$ is continuous at $t \neq t_{i}, u\left(t_{i}^{-}\right)=u\left(t_{i}\right)$ and $u\left(t_{i}^{+}\right)$exists for all $i=1,2, \ldots, n$. It is clear that PC endowed with the norm $\|x\|_{\mathrm{PC}}=\sup _{t \in J}\|x(t)\|$ is a Banach space. For any $x \in \mathrm{PC}$

$$
\tilde{x}_{i}(t)= \begin{cases}x(t), & t \in\left(t_{i}, t_{i+1}\right] \\ x\left(t_{i}^{+}\right), & t=t_{i}, \quad i=1,2, \ldots, n .\end{cases}
$$

So, $\tilde{x} \in C\left(\left[t_{i}, t_{i+1}\right], X\right)$.

Lemma 6 (see [31]). (1) If $W \subset P C([a, b] ; X)$ is bounded, then $\chi(W(t)) \leq \chi_{P C}(W)$ for any $t \in[a, b]$ where $W(t)=\{u(t): u \in$ $W\} \subset X$.

(2) If $W$ is piecewise equicontinuous on $[a, b]$, then $\chi(W(t))$ is piecewise continuous for $t \in[a, b]$, and

$$
\chi_{P C}(W)=\sup \{\chi(W(t)), t \in[a, b]\} .
$$

(3) If $W \subset P C([a, b] ; X)$ is bounded and piecewise equicontinuous, then $\chi(W(t))$ is piecewise continuous for $t \epsilon$ $[a, b]$ and

$$
\chi\left(\int_{a}^{t} W(s) d s\right) \leq \int_{a}^{t} \chi(W(s)) d s \quad t \in[a, b] .
$$

Lemma 7 (see [35]). If the semigroup $S(t)$ is equicontinuous and $\eta \in L\left([0, a] ; R^{+}\right)$, then the $\operatorname{set}\left\{\int_{0}^{t} S(t-s) u(s) d s:\|u(s)\| \leq\right.$ $\eta(s)$ for a.e $s \in[0, a]\}$ is equicontinuous for $t \in[0, b]$.

\section{Existence and Uniqueness of Mild Solution}

We define mild solution of problem (1) as follows.

Definition 8. A function $x:(-\infty, a] \rightarrow X$ is a mild solution of the problem (1) if $x_{0}=\phi ;\left.x(\cdot)\right|_{[0, a]} \in \mathrm{PC}(X), x(t)=$ $J_{i}^{1}\left(t, x_{t}\right) \forall t \in\left(t_{i}, s_{i}\right] i=1, \ldots, n$, and

$$
\begin{aligned}
& x(t)= C(t) \phi(0)+S(t)[z+g(0, \phi)] \\
&-\int_{0}^{t} C(t-s) g\left(s, x_{s}\right) d s \\
&+\int_{0}^{t} S(t-s) f\left(s, x_{\rho\left(s, x_{s}\right)}\right) d s, \\
& t \in\left[0, t_{1}\right], \\
& x(t)=C\left(t-s_{i}\right) J_{i}^{1}\left(s_{i}, x_{s_{i}}\right) \\
&+ S\left(t-s_{i}\right)\left(J_{i}^{2}\left(s_{i}, x_{s_{i}}\right)+g\left(s_{i}, x_{s_{i}}\right)\right) \\
&-\int_{s_{i}}^{t} C(t-s) g\left(s, x_{s}\right) d s \\
&+\int_{s_{i}}^{t} S(t-s) f\left(s, x_{\rho\left(s, x_{s}\right)}\right) d s \\
& \text { for } t \in\left[s_{i}, t_{i+1}\right] \quad i=1, \ldots, n .
\end{aligned}
$$

To prove our result we always assume $\rho: J \times \mathfrak{B} \rightarrow$ $(-\infty, a]$ is a continuous function. The following hypotheses are used.

$\left(\mathrm{H}_{\phi}\right)$ The function $t \rightarrow \phi_{t}$ is continuous from $\mathbb{R}\left(\rho^{-}\right)=$ $\{\rho(s, \psi): \rho(s, \psi) \leq 0\}$ into $\mathfrak{B}$ and there exists a continuous bounded function $J^{\phi}: \mathbb{R}\left(\rho^{-}\right) \rightarrow(0, \infty)$ such that $\left\|\phi_{t}\right\|_{\mathfrak{B}} \leq J^{\phi}(t)\|\phi\|_{\mathfrak{B}}$ for every $t \in \mathbb{R}\left(\rho^{-}\right)$.

(Hf) $f: J \times \mathfrak{B} \rightarrow X$ satisfies the following.

(1) For every $x:(-\infty, a] \rightarrow X, x_{0} \in \mathfrak{B}$ and $\left.x\right|_{J} \in \mathrm{PC}$, the function $f(\cdot, \psi): J \rightarrow X$ is strongly measurable for every $\psi \in \mathfrak{B}$ and $f(\cdot, t)$ is continuous for a.e. $t \in J$.

(2) There exists an integrable function $\alpha: J \rightarrow$ $[0,+\infty)$ and a monotone continuous nondecreasing function $\Omega:[0,+\infty) \rightarrow(0,+\infty)$ such that $\|f(t, v)\| \leq \alpha(t) \Omega\left(\|v\|_{\mathfrak{B}}\right) \forall t \in J$ and $v \in \mathfrak{B}$.

(3) There exists an integrable function $\eta: J \rightarrow[0, \infty)$ such that $\chi(S(s) f(t, D)) \leq$ $\eta(t) \sup _{-\infty<\theta<0} \chi(D(\theta))$ for a.e. $s, t, \epsilon J$, where $D(\theta)=\{v(\theta): v \in D\}$.

(Hg) The function $g(\cdot)$ is continuous $\forall t, v \in J \times \mathfrak{B}$ and $g(t, \cdot)$ is Lipschitz continuous such that there exists positive constant $L_{g}$ such that

$$
\begin{array}{r}
\left\|g\left(t, v_{1}\right)-g\left(t, v_{2}\right)\right\| \leq L_{g}\left\|v_{1}-v_{2}\right\|_{\mathfrak{B}}, \\
\left(t, v_{i}\right) \in J \times \mathfrak{B}, \quad i=1,2 .
\end{array}
$$


(HJ)

(1) There exist positive constants $c_{i}^{1}, c_{i}^{2}, d_{i}^{1}, d_{i}^{2}$ such that $\left\|J_{i}^{1}(t, v)\right\| \leq c_{i}^{1}\|v\|_{\mathfrak{B}}+c_{i}^{2}$ and $\left\|J_{i}^{2}(t, v)\right\| \leq$ $d_{i}^{1}\|v\|+d_{i}^{2}$.

(2) $\left\|J_{i}^{j}(t, u)-J_{i}^{j}(t, v)\right\| \leq L_{J_{i}^{j}}\|u-v\|_{\mathfrak{B}}$ for all $u, v \in$ $\mathfrak{B} i=1, \ldots, n, j=1,2$.

(1) $K_{a}\left(N a L_{g}+\widetilde{N} \int_{0}^{a} \alpha(s) d s \lim _{\tau \rightarrow \infty}\right.$ $\left.\sup (\Omega(\tau) / \tau)+\sum_{i=1}^{n}\left(N c_{i}^{1}+\widetilde{N}\left(d_{i}^{1}+L_{g}\right)\right)\right)+$ $\sum_{i=1}^{n} c_{i}^{1} K_{a}<1$

(2) $\left(K_{a} N L_{g} a+\sum_{i=1}^{n}\left\{N L_{J_{i}^{1}}+\widetilde{N}\left(L_{J_{i}^{2}}+L_{g}\right)\right\} K_{a}+\right.$ $\left.\sum_{i=0}^{n} \int_{s_{i}}^{t} \eta(s) d s\right)+\sum_{i=1}^{n}\left\{L_{J_{i}^{1}}\right\} K_{a}<1$.

(H1)

(1) $\quad K_{a}\left(N a L_{g}+\widetilde{N} \int_{0}^{a} \alpha(s) d s \lim _{\tau \rightarrow \infty}\right.$ $\left.\sup (\Omega(\tau) / \tau)+\sum_{i=1}^{n}\left(N c_{i}^{1}+\widetilde{N}\left(d_{i}^{1}+L_{g}\right)\right)\right)+$ $\sum_{i=1}^{n} c_{i}^{1} K_{a}<1$

(2) $\left(K_{a} N L_{g} a+\sum_{i=1}^{n}\left\{N L_{J_{i}^{1}}+\widetilde{N}\left(L_{J_{i}^{2}}+L_{g}\right)\right\} K_{a}+\right.$ $\left.\sum_{i=0}^{n} \int_{s_{i}}^{t} \eta(s) d s\right)+\sum_{i=1}^{n}\left\{L_{J_{i}^{1}}\right\} K_{a}<1$.

Lemma 9 (see [9]). If $y:(-\infty, a] \rightarrow X$ is a function such that $y_{0}=\phi$ and $\left.y\right|_{J} \in P C(X)$ then

$$
\begin{aligned}
&\left\|y_{\rho\left(s, y_{s}\right)}\right\|_{\mathfrak{B}} \leq\left(M_{a}+\widetilde{J^{\phi}}\right)\|\phi\|_{\mathfrak{B}} \\
&+K_{a} \sup \{\|y(\theta)\| ; \theta \in[0, \max \{0, s\}]\}, \\
& s \in \mathbb{R}\left(\rho^{-}\right) \cup[0, a],
\end{aligned}
$$

where $\widetilde{J^{\phi}}=\sup _{t \in \mathbb{R}\left(\rho^{-}\right)} J^{\phi}(t), M_{a}=\sup _{t \in J^{\prime}} M(t)$ and $K_{a}=$ $\max _{t \in J} K(t)$.

In this section : $(y-\infty, a] \rightarrow X$ is the function defined by $y_{0}=\phi$ and $y(t)=C(t) \phi(0)+S(t)(z+g(0, \phi))$ on $J_{1}=\left[0, t_{1}\right]$. Clearly $\left\|y_{t}\right\|_{\mathfrak{B}} \leq K_{a}\|y\|_{a}+M_{a}\|\phi\|_{\mathfrak{B}}$ where $\|y\|_{b}=$ $\sup _{0 \leq t \leq b}\|y(t)\|$.

Theorem 10. If the hypotheses $(\mathrm{Hf}),(\mathrm{Hg}),(\mathrm{HI}),(\mathrm{Hl})$ are satisfied, then the initial value problem (1) has at least one mild solution.

Proof. Let $S(a)$ be the space $S(a)=\left\{x:(-\infty, a] \rightarrow X \mid x_{0}=\right.$ $\left.0,\left.x\right|_{J} \in \mathrm{PC}\right\}$ endowed with supremum norm $\|\cdot\|_{a}$.
Let $\Gamma: S(a) \rightarrow S(a)$ be the map defined by $(\Gamma x)_{0}=0$ and $\Gamma=\sum_{i=1}^{n} \Gamma_{i}^{1}+\sum_{i=1}^{n} \Gamma_{i}^{2}:$

$$
\begin{aligned}
& \left(\Gamma_{i}^{1} x\right)(t) \\
& =\left\{\begin{array}{cc}
J_{i}^{1}\left(t, \bar{x}_{t}\right), & t \in\left(t_{i}, s_{i}\right] ; i=1, \ldots, n \\
C\left(t-s_{i}\right) J_{i}^{1}\left(s_{i}, \bar{x}_{s_{i}}\right) & \\
+S\left(t-s_{i}\right)\left(J_{i}^{2}\left(s_{i}, \bar{x}_{s_{i}}\right)\right. & \\
\left.+g\left(s_{i}, x_{s_{i}}\right)\right), & t \in\left(s_{i}, t_{i+1}\right] ; i=1, \ldots, n ;
\end{array}\right.
\end{aligned}
$$

$\left(\Gamma_{i}^{2} x\right)(t)$

$$
=\left\{\begin{array}{cl}
\int_{s_{i}}^{t} C(t-s) g\left(s, \overline{x_{s}}\right) d s & \\
\quad+\int_{s_{i}}^{t} S(t-s) & \\
\quad \times f\left(s, \bar{x}_{\rho\left(s, \overline{x_{s}}\right)}\right) d s, & t \in\left(s_{i}, t_{i+1}\right] ; i=0, \ldots, n \\
0, \quad & t \notin\left(s_{i}, t_{i+1}\right], i=0, \ldots, n,
\end{array}\right.
$$

where $\bar{x}_{0}=\phi$ and $\bar{x}=x+y$ on $J$. It is easy to see that

$$
\left\|\bar{x}_{t}\right\|_{\mathfrak{B}} \leq K_{a}\|y\|_{a}+M_{a}\|\phi\|_{\mathfrak{B}}+K_{a}\|x\|_{t}
$$

where $\|x\|_{t}=\sup _{0 \leq s \leq t}\|x(s)\|$.

$$
\left\|\bar{x}_{\rho\left(s, \bar{x}_{s}\right)}\right\|_{\mathfrak{B}} \leq k^{*}:=\left(M_{a}+\widetilde{J^{\phi}}\right)\|\phi\|_{\mathfrak{B}}+K_{a}\|y\|_{a}+K_{a}\|x\|_{a} .
$$

Thus $\Gamma$ is well defined and has values in $S(a)$. Also by axioms of phase space, the Lebesgue dominated convergence theorem, and the conditions ( $\mathrm{Hf}),(\mathrm{Hg})$ it can be shown that $\Gamma$ is continuous.

Step 1. There exists $k>0$ such that $\Gamma\left(B_{k}\right) \subset B_{k}$, where $B_{k}=$ $\left\{x \in S(a):\|x\|_{a} \leq k\right\}$. In fact, if we assume that the assertion is false, then for $k>0$ there exist $x_{k} \in B_{k}$ and $t_{k} \in\left(s_{i}, t_{i+1}\right]$ such that $k<\left\|\Gamma x_{k}\left(t_{k}\right)\right\|$ :

$$
\begin{aligned}
k \leq & \sum_{i=0}^{n}\left\|\Gamma_{i}^{2} x_{k}\left(t_{k}\right)\right\|+\sum_{i=1}^{n}\left\|\Gamma_{i}^{1} x_{k}\left(t_{k}\right)\right\| \\
\leq & \sum_{i=0}^{n} N \int_{s_{i}}^{t_{k}} L_{g}\left(\left\|\overline{x_{k s}}\right\|_{\mathcal{B}}+\|g(s, 0)\|\right) d s \\
& \left.+\sum_{i=1}^{n} \widetilde{N} \int_{s_{i}}^{t_{k}} \alpha(s) \Omega\left(\| \overline{x_{k \rho}\left(s, \overline{x_{k s}}\right.}\right) \|_{\mathcal{B}}\right) \\
& +\sum_{i=1}^{n} N\left(c_{i}^{1}\left\|\overline{x_{k s}}\right\|+c_{i}^{2}\right) \\
& +\sum_{i=1}^{n} \widetilde{N}\left(d_{i}^{1}\left\|\overline{x_{k s}}\right\|+d_{i}^{2}\right. \\
& \left.+L_{g}\left\|\overline{x_{k_{s_{i}}}}-0\right\|+\|g(s, 0)\|\right)
\end{aligned}
$$




$$
\begin{aligned}
& \leq \sum_{i=0}^{n} N \int_{s_{i}}^{t_{k}} L_{g}\left(K_{a}\|y\|_{a}+M_{a}\|\phi\|_{\mathfrak{B}}\right. \\
& \left.\quad+K_{a} k+\|g(s, 0)\|\right) d s \\
& +\sum_{i=0}^{n} \widetilde{N} \int_{s_{i}}^{t_{k}} \alpha(s) d s \\
& \quad \times \Omega\left(K_{a}\|y\|_{a}+\left(M_{a}+\widetilde{J} \phi\right)\|\phi\|_{\mathfrak{B}}+K_{a} k\right) \\
& +\sum_{i=1}^{n} N\left(c_{i}^{1}\left(K_{a}\|y\|_{a}+M_{a}\|\phi\|_{\mathfrak{B}}+K_{a} k\right)+c_{i}^{2}\right) \\
& +\widetilde{N}\left(d_{i}^{1}\left(K_{a}\|y\|_{a}+M_{a}\|\phi\|_{\mathfrak{B}}+K_{a} k\right)+d_{i}^{2}\right. \\
& \left.\quad+L_{g}\left(K_{a}\|y\|_{a}+M_{a}\|\phi\|_{\mathfrak{B}}+K_{a} k\right)+\|g(s, 0)\|\right) .
\end{aligned}
$$

Hence

$$
\begin{aligned}
& 1<\left(\widetilde{N} \int_{0}^{a} \alpha(s) d s\right. \\
& \quad \times \lim _{k \rightarrow \infty} \sup \frac{\Omega\left(K_{a}\|y\|_{a}+\left(M_{a}+\widetilde{J^{\phi}}\right)\|\phi\|_{\mathfrak{B}}+K_{a} k\right)}{k} \\
&\left.\quad+N a K_{a} L_{g}\right)+K_{a} \sum_{i=1}^{n}\left(N c_{i}^{1}+\widetilde{N}\left(d_{i}^{1}+L_{g}\right)\right) \\
& \leq K_{a}\left(N a L_{g}+\widetilde{N} \int_{0}^{a} \alpha(s) d s\right. \\
&\left.\quad \times \lim _{\tau \rightarrow \infty} \sup \frac{\Omega(\tau)}{\tau}+\sum_{i=1}^{n}\left(N c_{i}^{1}+\widetilde{N}\left(d_{i}^{1}+L_{g}\right)\right)\right)
\end{aligned}
$$

which is a contradiction to the hypothesis (H1). Similarly $(\Gamma x)(t)<k$, for $t_{k} \in\left(t_{i}, s_{i}\right] \forall i=1,2, \ldots, n$. Suppose on the contrary,

$$
\begin{aligned}
k & <\sum_{i=1}^{n}\left(\Gamma_{i}^{1} x_{k}\right)\left(t_{k}\right) \\
& =\sum_{i=1}^{n}\left\|J_{i}^{1}\left(t_{k}, \overline{x_{k}} t_{k}\right)\right\| \\
& \leq \sum_{i=1}^{n}\left\{c_{i}^{1}\left\|\overline{x_{k}}\right\|_{k} \|_{\mathfrak{B}}+c_{i}^{2}\right\} \\
& \leq \sum_{i=1}^{n}\left\{c_{i}^{1}\left(K_{a}\|y\|_{a}+M_{a}\|\phi\|_{\mathfrak{B}}+K_{a} k\right)+c_{i}^{2}\right\} .
\end{aligned}
$$

Hence,

$$
1<\sum_{i=1}^{n} c_{i}^{1} K_{a}
$$

which is a contradiction.
Step 2. To prove that $\Gamma$ is a $\chi$-contraction. Let $\Gamma=\sum_{i=1}^{n} \Gamma_{i}^{1}+$ $\sum_{i=0}^{n} \Gamma_{i}^{2}$ be split into $\Gamma=\sum_{i=1}^{n} \Gamma_{i}^{1}+\sum_{i=0}^{n}\left\{\Gamma_{i_{1}}^{2}+\Gamma_{i_{2}}^{2}\right\}$ for $t>0$

$$
\begin{gathered}
\Gamma_{i_{1}}^{2} x(t)=\int_{s_{i}}^{t} C(t-s) g\left(s, \overline{x_{s}}\right) d s, \\
\Gamma_{i_{2}}^{2} x(t)=\int_{s_{i}}^{t} S(t-s) f\left(s, \bar{x}_{\rho\left(s, \bar{x}_{s}\right)}\right) d s .
\end{gathered}
$$

For arbitrary $x_{1}, x_{2} \in \mathrm{B}_{k}$, and $t \in\left(s_{i}, t_{i+1}\right]$

$$
\begin{aligned}
& \sum_{i=0}^{n}\left\|\Gamma_{i_{1}}^{2} x_{1}(t)-\sum_{i=0}^{n} \Gamma_{i_{2}}^{1} x_{2}(t)\right\| \\
& \quad \leq \sum_{i=0}^{n}\left\|\int_{s_{i}}^{t} C(t-s)\left(g\left(s, x_{1_{s}}+y_{s}\right)-g\left(s, x_{2_{s}}+y_{s}\right)\right) d s\right\| \\
& \quad \leq \sum_{i=0}^{n} N L_{g} a\left\|x_{1_{t}}-x_{2_{t}}\right\|_{\mathcal{B}} \\
& \quad \leq K_{a} N L_{g} a\left\|x_{1}-x_{2}\right\|_{a} .
\end{aligned}
$$

So, $\Gamma_{i_{1}}^{2} \forall i=0, \ldots, n$ is Lipschitz continuous with Lipschitz constant $N L_{g} a K_{a}$.

For any $W \subset \Gamma_{i_{1}}^{2}\left(B_{k}\right), W$ is piecewise equicontinuous since $S(t)$ is equicontinuous. Hence from the fact that $\rho\left(s, \overline{x_{s}}\right) \leq$ $s, s \in[0, a]$ and Lemma 6 and $\chi_{\mathrm{PC}}(W)=\sup \{\chi(W(t)), t \in$ $J\}$ we have

$$
\begin{aligned}
& \chi\left(\sum_{i=0}^{n} \Gamma_{i_{1}}^{2} W(t)\right) \\
& \quad=\sum_{i=0}^{n} \chi\left(\int_{s_{i}}^{t} S(t-s) f\left(s, W_{\rho\left(s, \bar{x}_{s}\right)}+y_{s}\right) d s\right) \\
& \quad \leq \sum_{i=0}^{n} \int_{s_{i}}^{t} \eta(s) \sup _{-\infty<\theta \leq 0} \chi\left(W\left(\rho\left(s, \bar{x}_{s}\right)+\theta\right)+y(s+\theta)\right) d s \\
& \leq \sum_{i=0}^{n} \int_{s_{i}}^{t} \eta(s) \sup _{-\infty<\theta \leq 0} \chi(W(s+\theta)+y(s+\theta)) d s \\
& \quad \leq \sum_{i=0}^{n} \int_{s_{i}}^{t} \eta(s) \sup _{-\infty<\tau \leq 0} \chi W(\tau) d s \\
& \quad \leq \chi_{\mathrm{PC}}(W) \sum_{i=0}^{n} \int_{s_{i}}^{t} \eta(s) d s .
\end{aligned}
$$


For arbitrary $x_{1}, x_{2} \in B_{k}$ and $t \in\left(s_{i}, t_{i+1}\right]$

$$
\begin{aligned}
& \sum_{i=1}^{n}\left\|\left(\Gamma_{i}^{1} x_{1}\right)(t)-\sum_{i=1}^{n}\left(\Gamma_{i}^{1} x_{2}\right)(t)\right\| \\
& \leq \sum_{i=1}^{n}\left\{N L_{J_{i}^{1}}\left\|\bar{x}_{1_{s}}-\overline{x_{2} s_{i}}\right\|\right. \\
& \left.\quad+\widetilde{N}\left(L_{J_{i}^{2}}\left\|\bar{x}_{s_{i}}-\overline{x_{2}} s_{i}\right\|+L_{g}\left\|x_{2 s_{i}}-x_{1 s_{i}}\right\|\right)\right\} \\
& \leq \sum\left\{N L_{J_{i}^{1}}+\widetilde{N}\left(L_{J_{i}^{2}}+L_{g}\right)\right\}\left\|x_{1 s_{i}}+y_{s}-x_{2 s_{i}}-y_{s}\right\| \\
& \leq \sum\left\{N L_{J_{i}^{1}}+\widetilde{N}\left(L_{J_{i}^{2}}+L_{g}\right)\right\}\left\|x_{1 s_{i}}-x_{2 s_{i}}\right\|_{\mathcal{B}} \\
& \leq \sum\left\{N L_{J_{i}^{1}}+\widetilde{N}\left(L_{J_{i}^{2}}+L_{g}\right)\right\} K_{a}\left\|x_{1}-x_{2}\right\|_{a} .
\end{aligned}
$$

So, $\Gamma_{i}^{1} \forall i=1, \ldots, n$ is Lipschitz continuous with Lipschitz constant $\left(N L_{J_{i}^{1}}+\widetilde{N} L_{J_{i}^{2}}\right) K_{a}$.

For arbitrary $x_{1}, x_{2} \in B_{k}$ and $t \in\left(t_{i}, s_{i}\right]$,

$$
\begin{aligned}
\sum_{i=1}^{n}\left\|\left(\Gamma_{i}^{1} x_{1}\right)(t)-\sum_{i=1}^{n}\left(\Gamma_{i}^{1} x_{2}\right)(t)\right\| & \leq \sum_{i=1}^{n} L_{J_{i}^{1}}\left\|x_{1_{t}}-x_{2_{t}}\right\|_{\mathfrak{B}} \\
& \leq \sum_{i=1}^{n} K_{a} L_{J_{i}^{1}}\left\|x_{1}-x_{2}\right\|_{a} .
\end{aligned}
$$

For each bounded set $W \in \mathrm{PC}(J ; X)$ and $t \in\left(s_{i}, t_{i+1}\right], \forall i=$ $0, \ldots, n$ we have

$$
\begin{aligned}
\chi_{\mathrm{PC}}(\Gamma W) \leq & \sum_{i=1}^{n} \chi_{\mathrm{PC}}\left(\Gamma_{i}^{1} W\right)+\sum_{i=0}^{n} \chi_{\mathrm{PC}}\left(\Gamma_{i_{1}}^{2} W+\Gamma_{i_{2}}^{2} W\right) \\
\leq & \left(K_{a} N L_{g} a+\sum\left\{N L_{J_{i}^{1}}+\widetilde{N}\left(L_{J_{i}^{2}}+L_{g}\right)\right\} K_{a}\right. \\
& \left.+\sum_{i=0}^{n} \int_{s_{i}}^{t} \eta(s) d s\right) \chi_{\mathrm{PC}}(W) .
\end{aligned}
$$

For each bounded set $W \in \mathrm{PC}(J ; X)$ and $t \in\left(t_{i}, s_{i}\right] \quad \forall i=$ $1,2, \ldots, n$ we have

$$
\begin{aligned}
\chi_{\mathrm{PC}}(\Gamma W) & \leq \sum_{i=1}^{n} \chi_{\mathrm{PC}}\left(\Gamma_{i}^{1} W\right)+\sum_{i=0}^{n} \chi_{\mathrm{PC}}\left(\Gamma_{i_{1}}^{2} W+\Gamma_{i_{2}}^{2} W\right) \\
& \leq\left(\sum_{i=1}^{n}\left\{L_{J_{i}^{1}}\right\} K_{a}+0+0\right) \chi_{\mathrm{PC}}(W) .
\end{aligned}
$$

Therefore, $\Gamma$ is a $\chi$-contraction. So, by Darbo-Sadovskii fixed point theorem we conclude that $\Gamma$ has a fixed point in $S(a)$. hence, $z=x+y$ is a mild solution of (1).

\section{Approximate Controllability}

In this section the approximate controllability of the control system (1) without the impulsive conditions is studied. We consider

$$
\begin{gathered}
\frac{d}{d t}\left(x^{\prime}(t)+g\left(t, x_{t}\right)\right)=A x(t)+f\left(t, x_{\rho\left(t, x_{t}\right)}\right)+B u(t), \\
t \in J=[0, a], \\
x_{0}=\phi \in \mathfrak{B}, \quad x^{\prime}(0)=w \in X,
\end{gathered}
$$

where $A$ is the infinitesimal generator of a strongly continuous cosine family $\{C(t): t \in \mathbb{R}\}$ of bounded linear operators on a Hilbert space $X$. The history valued function $x_{t}:(-\infty, 0] \rightarrow X, x_{t}(\theta)=x(t+\theta)$ belongs to some abstract phase space $\mathfrak{B}$ defined axiomatically; $g, f$ are appropriate functions. $B$ is a bounded linear operator on a Hilbert space $U$. We define mild solution of problem (24) as follows.

Definition 11. A function $x:(-\infty, a] \rightarrow X$ is a mild solution of the problem (24) if $x_{0}=\phi ;\left.x(\cdot)\right|_{[0, a]} \in C(J, X)$, the functions $f\left(s, x_{\rho\left(s, x_{s}\right)}\right)$ and $g\left(s, x_{s}\right)$ are integrable and the integral equation is satisfied:

$$
\begin{aligned}
x(t)= & C(t) \phi(0)+S(t)[w+g(0, \phi)] \\
& -\int_{0}^{t} C(t-s) g\left(s, x_{s}\right) d s \\
& +\int_{0}^{t} S(t-s)\left[f\left(s, x_{\rho}\left(s, x_{s}\right)\right)+B u(s)\right] d s, \\
& t \in[0, a] .
\end{aligned}
$$

Lemma 12 (see [11]). Under the assumption that $h:[0, a] \rightarrow$ $X$ is an integrable function, such that

$$
\begin{gathered}
x^{\prime \prime}(t)=A x(t)+h(t), \quad t \in J, \\
x(0)=x^{0} \\
x^{\prime}(0)=x^{1}
\end{gathered}
$$

and $h$ is a function continuously differentiable, then

$$
\int_{0}^{t} C(t-s) h(s) d s=S(t) h(0)+\int_{0}^{t} S(t-s) h^{\prime}(s) d s .
$$

Set $a:=T$.

Definition 13. The set given by $\mathscr{R}_{T}(f)=\{x(T) \in X: x$ is the mild solution of (24)\} is called reachable set of the system (24). $\mathscr{R}_{T}(0)$ is the reachable set of the corresponding linear control system (31).

Definition 14. The system (24) is said to be approximately controllable on $[0, T]$ if $\mathscr{R}_{T}(f)$ is dense in $X$. The corresponding linear system is approximately controllable if $\mathscr{R}(0)$ is dense in $X$. 
Lemma 15. Let $X$ be Hilbert space and $X_{1}, X_{2}$ closed subspaces such that $X=X_{1}+X_{2}$. Then there exists a bounded linear operator $P: X \rightarrow X_{2}$ such that for each $x \in X, x=$ $x-P x \in X_{1}$ and $\left\|x_{1}\right\|=\min \left\{\|y\|: y \in X_{1},(1-Q)(y)=\right.$ $(1-Q)(x)\}$ where $Q$ denotes the orthogonal projection on $X_{2}$.

Let us define a continuous linear operator $\mathfrak{Q}$ : $\mathscr{L}_{2}([0, T] ; X) \rightarrow C([0, T] ; X)$ as

$$
\mathfrak{\Omega} p=\int_{0}^{T} S(T-s) p(s) d s, \quad p \in \mathscr{L}_{2}([0, T] ; X) .
$$

Let us denote the kernel of the operator $\mathfrak{Q}$ by $N$ which is a closed subspace of $\mathscr{L}_{2}([0, T] ; X)$. Let $N_{0}^{\perp}$ denote the corresponding orthogonal subspace of $\mathscr{L}_{2}([0, T] ; X)$. Let $\mathfrak{P}$ be a projection on $\mathscr{L}_{2}([0, T] ; X)$ with range $N_{0}^{\perp}$. Let $\overline{R(B)}$ denote the closure of the range of operator $B$. The following hypothesis is required to prove the approximate controllability

(HR) $\forall \epsilon>0$ and $p(\cdot) \in \mathscr{L}_{2}([0, T] ; X), \exists u(\cdot) \in U$ such that $\|\mathfrak{Q} p-\mathfrak{\Omega} B u\|_{X}<\epsilon$.

It is easily seen that hypothesis (HR) is equivalent to the $\mathscr{L}_{2}([0, T] ; X)=\overline{R(B)}+N_{0}$ or $\mathfrak{P ( B )}=N_{0}^{\perp}$. Theorem 16 shows that (HR) implies approximate controllability of the system (29). It is also known that approximate controllability of (31) implies $\mathscr{L}_{2}([0, T] ; X)=\overline{R(B)+N_{0}}$. Hence the closeness of the product space implies that (HR) is equivalent to approximate controllability of (29).

Theorem 16. If the assumptions $(\mathrm{Hg})$ and $(\mathrm{HR})$ hold then the corresponding neutral system

$$
\begin{gathered}
\frac{d\left(x^{\prime}(t)+g\left(t, x_{t}\right)\right)}{d t \quad}=A x(t)+B u(t), \quad t \in J, \\
x(0)=\phi(0), \\
x^{\prime}(0)=w
\end{gathered}
$$

with $f \equiv 0$ is approximately controllable.

Proof. It is sufficient to prove that $D(A) \subset \overline{\mathscr{R}_{T}(0)}$ since $D(A)$ is dense in $X$. Let $h(T, \phi)=C(t) \phi(0)+S(t)[w+g(0, \phi(0))]-$ $\int_{0}^{T} C(t-s) g\left(s, x_{s}\right) d s$ for any chosen $\xi \in D(A)$, then $\xi-$ $h(T, \phi) \in D(A)$. It can be easily seen from Lemma 12 and [28] that there exists some $p \in C^{1}([0, T] ; X)$ such that

$$
\eta=\xi-h(T, \phi)=\int_{0}^{T} S(T-s) p(s) d s .
$$

By hypothesis (HR) there exists a control function $u(\cdot) \epsilon$ $\mathscr{L}_{2}([0, T] ; U)$ such that $\|\eta-\mathfrak{Q B u}\|<\epsilon$. As $\epsilon$ is arbitrary it implies that $K_{T}(0) \subset D(A)$. Since the $D(A)$ is dense in $X, K_{T}(0)$ is dense in $X$. Hence the neutral system with $f \equiv 0$ is approximately controllable.
We state the corresponding linear control system

$$
\begin{gathered}
x^{\prime \prime}(t)=A x(t)+B u(t), \quad t \in J, \\
x(0)=x^{0}, \\
x^{\prime}(0)=x^{1} .
\end{gathered}
$$

Both exact and approximate controllability of the above system are studied extensively in $[33,38]$ and so forth.

Assume that $f, g$ satisfy the following conditions with $\mu_{f}, \mu_{g}, \nu_{f}, \nu_{g} \in L^{2}(J)$. For a fixed $\phi \in \mathfrak{B}$ and $x \in C(J, X)$ such that $x(0)=\phi(0)$, we define maps $F, G: C_{0}(J, X) \rightarrow L^{2}(J, X)$ by $F(z)(t)=f\left(t, z_{t}+x_{t}\right)$ and $G(z)(t)=g\left(t, z_{t}+x_{t}\right)$. Here $x_{t}(\theta)=x(t+\theta)$, for $t+\theta \geq 0$ and $x_{t}(\theta)=\phi(t+\theta)$ for $t+\theta \leq 0$ and $z_{t}(\theta)=z(t+\theta)$ for $t+\theta \geq 0$ and $z_{t}(\theta)=0$ for $t+\theta \leq 0$. Clearly, $F, G$ are continuous maps.

(C1) The function $F(t, \cdot): \mathfrak{B} \rightarrow X$ is continuous for almost all $t \in I$ and $F(\cdot, z): J \rightarrow X$ is strongly measurable, $\forall z \in \mathfrak{B}$.

(C2) There exists integrable functions $\mu_{F}, \nu_{F}: I \rightarrow$ $[0, \infty)$ and a continuous nondecreasing function $W_{F}:[0, \infty) \rightarrow(0, \infty)$ such that $\|F(t, z)\|_{2} \leq$ $\mu_{F}(t) W_{F}\left(\|z\|_{1}\right)+v_{F}(t),(t, z) \in J \times \mathfrak{B}$

(C3) The function $f(\cdot)$ is continuous $\forall t, v \in J \times \mathfrak{B}$ and $f(t, \cdot)$ is Lipschitz continuous such that there exists positive constant $L_{f}$ such that

$$
\begin{array}{r}
\left\|f\left(t, v_{1}\right)-f\left(t, v_{2}\right)\right\| \leq L_{f}\left\|v_{1}-v_{2}\right\|_{\mathfrak{B}}, \\
\left(t, v_{i}\right) \in J \times \mathfrak{B}, \quad i=1,2 .
\end{array}
$$

The above same conditions also hold for $G$.

Also, $y:(-\infty, a] \rightarrow X$ is the function defined by $y_{0}=\phi$ and $y(t)=C(t) \phi(0)+S(t)(z+g(0, \phi))$ on $J$. Clearly $\left\|y_{t}\right\|_{\mathfrak{B}} \leq$ $K_{a}\|y\|_{a}+M_{a}\|\phi\|_{\mathfrak{B}}$ where $\|y\|_{b}=\sup _{0 \leq t \leq b}\|y(t)\|$.

The operators $\Lambda_{i}: L^{2}(J, X) \rightarrow X \quad i=1,2$ are defined as

$$
\begin{aligned}
& \Lambda_{1} x(t)=\int_{0}^{a} S(t-s) x(s) d s, \\
& \Lambda_{2} x(t)=\int_{0}^{a} C(t-s) x(s) d s .
\end{aligned}
$$

Clearly $\Lambda_{i}$ are bounded linear operators. We set $\mathfrak{N}_{i}=$ $\operatorname{ker}\left(\Lambda_{i}\right), \Lambda=\left(\Lambda_{1}, \Lambda_{2}\right)$ and $\mathfrak{N}=\operatorname{ker}(\Lambda)$. Let $C_{0}(J, X)$ denote the space consisting of continuous functions $x: J \rightarrow X$ such that $x(0)=0$, endowed with the norm of uniform convergence. Let $J_{i}: L^{2}(J, X) \rightarrow C_{0}(J, X), i=1,2$ be maps defined as follows:

$$
\begin{aligned}
& J_{1} x(t)=\int_{0}^{t} S(t-s) x(s) d s, \\
& J_{2} x(t)=\int_{0}^{t} C(t-s) x(s) d s .
\end{aligned}
$$

So, $J_{i} x(a)=\Lambda_{i}(x), i=1,2$. 
As a continuation of coauthor Sukavanam's work [19] and from hypothesis $(B 1)$ in [39] we assume that $L^{2}(J, X)=\mathfrak{N}_{i}+$ $\overline{R(B)}, i=1,2$.

By using Lemma 15 we denote $P_{i}$ the map associated to this decomposition and construct $X_{2}=\mathfrak{N}_{i}$ and $X_{1}=\overline{R(B)}$. Also set $c_{i}=\left\|P_{i}\right\|$.

We introduce the space

$$
Z=\left\{z \in C_{0}(J, X): z=J_{1}\left(n_{1}\right)+J_{2}\left(n_{2}\right), n_{i} \in \mathfrak{N}_{i}, i=1,2\right\}
$$

and we define the map $\Gamma: \bar{Z} \rightarrow C_{0}(J, X)$ by

$$
\Gamma=J_{1} \circ P_{1} \circ F-J_{2} \circ P_{2} \circ G .
$$

Lemma 17. If the hypothesis $\left(\mathrm{H}_{\phi}\right)-(\mathrm{Hg})$ and conditions $(\mathrm{Cl})-$ (C2) hold for $f$, g and $a K_{a}\left(c_{1} \tilde{N} L_{f}+c_{2} N L_{g}\right)<\sqrt{2}$ then $\Gamma$ has a fixed point.

Proof. For $z^{1}, z^{2} \in \bar{Z}$ let $\Delta f(s)=f\left(s, z_{\rho\left(s, z^{2}(s)\right)}^{2}+x_{\rho(s, x(s))}\right)-$ $f\left(s, z_{\rho\left(s, z^{1}(s)\right)}^{1}+x_{\rho(s, x(s))}\right)$ and $\Delta g(s)=g\left(s, z_{s}^{2}+x_{s}\right)-f\left(s, z_{s}^{1}+\right.$ $\left.x_{s}\right) . \forall 0 \leq t \leq a$

$$
\begin{aligned}
& \left\|\left(\Gamma z^{2}-\Gamma z^{2}\right)(t)\right\| \\
& \leq\left\|\int_{0}^{t} S(t-s)\left[P_{1}(\Delta f)\right](s) d s\right\| \\
& \quad+\left\|\int_{0}^{t} C(t-s)\left[P_{2}(\Delta g)\right](s) d s\right\| \\
& \leq \widetilde{N} \int_{0}^{t}\left\|\left[P_{1}(\Delta f)\right](s)\right\| d s+N \int_{0}^{t}\left\|\left[P_{2}(\Delta g)\right](s)\right\| d s \\
& \leq \widetilde{N} t^{1 / 2} c_{1}\|\Delta f\|_{2}+N t^{1 / 2} c_{2}\|\Delta g\|_{2} .
\end{aligned}
$$

Now

$$
\begin{aligned}
\|\Delta f\|_{2}^{2}= & \int_{0}^{a} \| f\left(s, z_{\rho\left(s, z^{2}(s)\right)}^{2}+x_{\rho(s, x(s))}\right) \\
& \quad-f\left(s, z_{\rho\left(s, z^{1}(s)\right)}^{1}+x_{\rho(s, x(s))}\right) \|^{2} d s \\
\leq & L_{f}^{2} \int_{0}^{a}\left\|z_{\rho\left(s, z^{2}(s)\right)}^{2}-z_{\rho\left(s, z^{1}(s)\right)}^{1}\right\|_{\mathfrak{B}}^{2} d s \\
\leq & L_{f}^{2} \int_{0}^{a}\left\|z_{s}^{2}-z_{s}^{1}\right\|_{\mathfrak{B}}^{2} d s \\
\leq & a L_{f}^{2} K_{a}^{2}\left\|z^{2}-z^{1}\right\|_{\infty}^{2} d s .
\end{aligned}
$$

Similarly we find for $g$. So,

$$
\left\|\left(\Gamma z^{2}-\Gamma z^{1}\right)(t)\right\| \leq b t^{1 / 2}\left\|z^{2}-z^{1}\right\|_{\infty},
$$

where $b=a^{1 / 2} K_{a}\left(c_{1} \widetilde{N} L_{f}+c_{2} N L_{g}\right)$. Repeating this we get

$$
\left\|\left(\Gamma^{n} z^{2}-\Gamma^{n} z^{1}\right)(t)\right\|_{\infty} \leq \frac{\left(b t^{1 / 2}\right)^{n}}{2^{(n-1) /(2 n)}}\left\|z^{2}-z^{1}\right\|_{\infty} .
$$

As $b=a K_{a}\left(c_{1} \widetilde{N} L_{f}+c_{2} N L_{g}\right)<\sqrt{2}$ and $2^{(n-1) / 2 n} \rightarrow$ $\sqrt{2}$ as $n \rightarrow \infty$, the map $\Gamma^{n}$ is a contraction for $n$ sufficiently large and therefore $\Gamma$ has a fixed point.
Theorem 18. If the associated linear control system (31) is approximately controllable on $J$, the space $L^{2}([0, a], X)=\mathfrak{N}_{i}+$ $\overline{R(B)}, i=1,2$ and condition of the preceding Lemma 17 hold then the semilinear control system (24) with state dependent delay is approximately controllable on $\mathrm{J}$.

Proof. Assume $x(\cdot)$ to be the mild solution and $u(\cdot)$ to be an admissible control function of system (31) with initial conditions $x(0)=\phi(0)$ and $x^{\prime}(0)=w+g(0, \phi)$. Let $z$ be the fixed point of $\Gamma$. So, $z(0)=0$ and $z(a)=\Lambda_{1}\left(P_{1}(F(z))\right)-$ $\Lambda_{2}\left(P_{2}(G(z))\right)=0$. By Lemma 12 we can split the functions $F(z), G(z)$ with respect to the decomposition $L^{2}(J, X)=\mathfrak{N}_{i}+$ $\overline{R(B)} i=1,2$, respectively, by setting $q_{1}=F(z)-P_{1}(F(z))$ and $q_{2}=G(z)-P_{2}(G(z))$. We define the function $y(t)=z(t)+x(t)$ for $t \in J$ and $y_{0}=\phi$. So, $x(a)=y(a)$. Thus by the properties of $x$ and $z$

$$
\begin{aligned}
y(t) & \\
= & \int_{0}^{t} S(t-s)\left(f\left(s, y_{\rho(s, y(s))}\right)-q_{1}(s)+B u(s)\right) d s \\
& -\int_{0}^{t} C(t-s)\left(g\left(s, y_{s}\right)-q_{2}(s)\right) d s \\
& +C(t) x(0)+S(t) x^{\prime}(0) .
\end{aligned}
$$

As $C_{0}^{1}(J, U)$ is dense in $L^{2}(J, U)$ we can choose a sequence $v_{n}^{1} \epsilon$ $L^{2}(J, U)$ and a sequence $v_{n}^{2} \in L^{2}(J, X)$ such that $B v_{n}^{1} \rightarrow q_{1}$ and $B v_{n}^{2} \rightarrow q_{2}$ as $n \rightarrow \infty$. By Lemma 15 we get

$$
\begin{aligned}
y^{n}(t) & \\
= & \int_{0}^{t} S(t-s)\left(f\left(s, y_{\rho(s, y(s))}^{n}\right)-B v_{n}^{1}(s)+B u(s)\right) d s \\
& -\int_{0}^{t} C(t-s)\left(g\left(s, y_{s}^{n}\right)-B v_{n}^{2}(s)\right) d s \\
& +C(t) \phi(0)+S(t)(w-g(0, \phi)) \\
= & \int_{0}^{t} S(t-s)\left(f\left(s, y_{\rho(s, y(s))}^{n}\right)-B v_{n}^{1}(s)\right. \\
& \left.+B \frac{d}{d s} v_{n}^{2}(s)+B u(s)\right) d s \\
& -\int_{0}^{t} C(t-s) g\left(s, y_{s}^{n}\right) d s+C(t) \phi(0) \\
& +S(t)(w+g(0, \phi)) .
\end{aligned}
$$

Hence by Definition 11 and the last expression we conclude that $y^{n}$ is the mild solution of the following equation:

$$
\begin{gathered}
\frac{d}{d t}\left(y^{\prime}(t)+g\left(t, x_{t}\right)\right) \\
=A y(t)+f\left(t, y_{\rho(t, y(t))}\right)+B\left(-v_{n}^{1}(t)+\frac{d}{d t} v_{n}^{2}(t)+u(t)\right) \\
x(0)=\phi \in \mathfrak{B} \quad x^{\prime}(0)=w .
\end{gathered}
$$


Hence $y^{n}(a) \in \mathscr{R}_{T}(a, f, g, \phi, w)$. Since the solution map is generally continuous, $y^{n} \rightarrow y$ as $n \rightarrow \infty$. Thus $y(a) \in$ $\mathscr{R}_{T}(a, f, g, \phi, w)$. Therefore $\mathscr{R}_{T}(0)(a, \phi(0), w+g(0, \phi)) \quad c$ $\overline{\mathscr{R}_{T}(a, f, g, \phi, w)}$, which means $\mathscr{R}_{T}(a, f, g, \phi, w)$ is dense in $X$. Thus the system (1) is controllable.

\section{Examples}

Example 1. In this section we discuss a partial differential equation applying the abstract results of this paper. In this application, $\mathfrak{B}$ is the phase space $C_{0} \times L^{2}(h, X)$ (see [10]).

Consider the second order neutral differential equation:

$$
\begin{aligned}
& \frac{\partial}{\partial t}\left(\frac{\partial u(t, \xi)}{\partial t}+\int_{-\infty}^{t} \int_{0}^{\pi} b(t-s, \eta, \xi) u(s, \eta) d \eta d s\right) \\
& =\frac{\partial^{2} u(t, \xi)}{\partial \xi^{2}} \\
& +\int_{-\infty}^{t} a(t-s) u\left(s-\rho_{1}(t) \rho_{2}(\|u(t)\|), \xi\right) d s, \\
& t \in\left(s_{i}, t_{i+1}\right], \quad i=0, \ldots, n, \xi \in[0, \pi], \\
& u(t, 0)=u(t, \pi)=0, \quad t \in[0, a], \\
& u(\tau, \xi)=\phi(\tau, \xi) \quad \tau \leq 0,0 \leq \xi \leq \pi, \\
& u^{\prime}(\tau, \xi)=\omega(t, \xi) \quad \tau \leq 0,0 \leq \xi \leq \pi, \\
& u(t)(\xi)=\int_{\infty}^{t_{i}} a_{i}^{1}\left(t_{i}-s\right) u(s, \xi) d s \\
& t \in\left(t_{i}, s_{i}\right], \quad i=1,2, \ldots, n, \\
& u^{\prime}(t)(\xi)=\int_{\infty}^{t_{i}} a_{i}^{2}\left(t_{i}-s\right) u(s, \xi) d s \\
& t \in\left(t_{i}, s_{i}\right], \quad i=1,2, \ldots, n,
\end{aligned}
$$

where $\phi \in C_{0} \times L^{2}(h, X), 0<t_{1}<, \ldots, t_{n}<a$. For $y \in D(A)$, $y=\sum_{n=1}^{\infty}<y, \phi_{n}>\phi_{n}$ and $A y=-\sum_{n=1}^{\infty} n^{2}<y, n>\phi_{n}$, where $\phi_{n}(x)=\sqrt{2 / \pi} \sin n x, 0 \leq x \leq \pi, n=1,2,3, \ldots$ is the eigenfunction corresponding to the eigenvalue $\lambda_{n}=-n^{2}$ of the operator $A . \phi_{n}$ is an orthonormal base. $A$ will generate the operators $S(t), C(t)$ such that $S(t) y=\sum_{n=1}^{\infty}((\sin (n t)) / n)<$ $y, \phi_{n}>\phi_{n}, n=1,2, \ldots \forall y \in X$, and the operator $C(t) y=$ $\sum_{n=1}^{\infty} \cos (n t)<y, \phi_{n}>\phi_{n}, n=1,2, \ldots \forall y \in X$. To find a solution to this problem we will assume that $h(\cdot)$ satisfies the conditions (g-5)-(g-7) in [34]. From Theorems 1.37 and 7.1.1 in [34] we conclude that $C_{a}((-\infty, 0], X)$ is continuously included in $\mathfrak{B}$. Let us suppose that the functions $\rho_{i}: \mathbb{R} \rightarrow$
$[0, \infty), a: \mathbb{R} \rightarrow \mathbb{R}$ are piecewise continuous. By defining maps $\rho, G, F:[0, a] \times \mathfrak{B} \rightarrow X$ by

$$
\begin{gathered}
\rho(t, \psi):=\rho_{1}(t) \rho_{2}(\|\psi(0)\|), \\
g(t, \psi)(\xi):=\int_{-\infty}^{0} \int_{0}^{\pi} b(s, v, \xi) \psi(s, v) d v d s, \\
f(t, \psi)(\xi):=\int_{-\infty}^{0} a(s) \psi(s, \xi) d s, \\
J_{i}^{j}(\psi)(\xi):=\int_{-\infty}^{0} a_{i}^{j}(s) \psi(s, \xi) d s \quad i=1, \ldots, n j=1,2
\end{gathered}
$$

the system (51) can be transformed into system (1). Assume that the following conditions hold:

(a) the functions $b(s, \eta, \xi), \partial b(s, \eta, \xi) / \partial \xi$ are measurable, $b(s, \eta, \pi)=b(s, \eta, 0)=0$ and

$$
\begin{aligned}
& L_{g} \\
& :=\max \left\{\left(\int_{0}^{\pi} \int_{-\infty}^{0} \int_{0}^{\pi} \frac{1}{h(s)}\left(\frac{\partial^{i} b(s, \eta, \xi)}{\partial \xi^{i}}\right)^{2} d \eta d s d \xi\right)^{1 / 2}:\right. \\
& \qquad i=0,1\} \\
& <\infty
\end{aligned}
$$

such that $\|g\|_{\mathscr{L}(X)} \leq L_{g}$.

(b) The function $F: \mathbb{R} \times \mathbb{R} \rightarrow \mathbb{R}$ is continuous and there is continuous function $L_{f}=\int_{-\infty}^{0}\left(a(s)^{2} / h(s)\right) d s<\infty$ and $\|F\|_{\mathscr{L}(X)} \leq L_{f}$.

(c) The functions $a_{i}^{j} \in C([0, \infty) ; \mathbb{R})$ and $L_{i}^{j} \quad:=$ $\left(\int_{-\infty}^{0}\left(\left(a_{i}^{j}(s)\right)^{2} / h(s)\right) d s\right)^{1 / 2}<\infty$ for all $i=$ $1,2, \ldots, n j=1,2$.

Moreover $g(t, \cdot), J_{i}^{j}, i=1, \ldots, n, j=1,2$ are bounded linear operators.

Hence by assumptions (a)-(c) and Theorem 10 it is ensured that mild solution to the problem (51) exists. 
Now let us consider a particular example from the point of view of an application:

$$
\begin{gathered}
\frac{\partial}{\partial t}\left(\frac{\partial u(t, \xi)}{\partial t}+\int_{-\infty}^{t} \int_{0}^{\pi} b(t-s, \eta, \xi) u(s, \eta) d \eta d s\right) \\
=\frac{\partial^{2} u(t, \xi)}{\partial \xi^{2}}+a(t) b(u(t-\mu(u(t, 0)), \xi)), \\
t \in\left(s_{i}, t_{i+1}\right], \quad i=0, \ldots, n, \xi \in[0, \pi], \\
u(t, 0)=u(t, \pi)=0, \quad t \in[0, a], \\
u(\tau, \xi)=\phi(\tau, \xi) \quad \tau \leq 0,0 \leq \xi \leq \pi, \\
u^{\prime}(\tau, \xi)=\omega(\tau, \xi) \quad \tau \leq 0,0 \leq \xi \leq \pi, \\
u(t)(\xi)=d_{i}^{1} \sin |u(t, \xi)|, \quad t \in\left(t_{i}, s_{i}\right], i=1,2, \ldots, n, \\
u^{\prime}(t)(\xi)=d_{i}^{2} \cos |u(t, \xi)|, \quad t \in\left(t_{i}, s_{i}\right], i=1,2, \ldots, n,
\end{gathered}
$$

where $\phi \in \mathfrak{B}=C_{h}^{0}(X)$. The functions $a: J \rightarrow \mathbb{R}, b: \mathbb{R} \times J \rightarrow$ $\mathbb{R}, \mu: \mathbb{R} \rightarrow \mathbb{R}^{+}$are piecewise continuous. We assume the existence of positive constants $b_{1}, b_{2}$ such that

$$
|b(t)| \leq b_{1}|t|+b_{2}, \quad \forall t \in \mathbb{R}
$$

If we define maps

$$
\begin{gathered}
f(t, \psi)(\xi)=a(t) b(\psi(0, \xi)), \\
\rho(t, \psi)=t-\mu(\psi(0,0)),
\end{gathered}
$$

and $g(t, \psi)(\xi)$ as in the problem (51) we can transform (47) into (1). Also a simple estimate shows that $\|f(t, \psi)\| \leq$ $a(t)\left[b_{1}\|\psi\|_{\mathfrak{B}}+b_{2} \pi^{1 / 2}\right] \forall(t, \psi) \in J \times \mathfrak{B}$.

Also if we define $J_{i}^{1}(t, u(t))=d_{i}^{1} \sin |u(t)|$ and $J_{i}^{2}=$ $d_{i}^{2} \cos |u(t)|$ for all $i=1, \ldots, n$ then the hypotheses $(\mathrm{HJ})$ can be easily proved. For instance,

$$
\begin{gathered}
\left\|J_{i}^{1}(t, u(t))\right\|=\left\|d_{i}^{1} \sin |u(t)|\right\| \leq d_{i}^{1}\|u(t)\|, \\
\left\|J_{i}^{1}\left(t, u_{1}(t)\right)-J_{i}^{1}\left(t, u_{2}(t)\right)\right\| \\
=\left\|d_{i}^{1} \sin \left|u_{1}(t)\right|-d_{i}^{1} \sin \left|u_{2}(t)\right|\right\| \\
\leq\left\|d_{i}^{1}\left[\left|u_{1}(t)\right|-\left|u_{2}(t)\right|\right]\right\| .
\end{gathered}
$$

Similarly it is easily seen for $J_{i}^{2}$. Now, if $\phi$ satisfies the hypothesis $\left(\mathrm{H}_{\phi}\right)$ then $\exists$ a mild solution of (47).
Example 2. Consider the second order neutral differential equation:

$$
\begin{gathered}
\frac{\partial}{\partial t}\left(\frac{\partial u(t, \xi)}{\partial t}+\int_{-\infty}^{t} \int_{0}^{\pi} b(t-s, \eta, \xi) u(s, \eta) d \eta d s\right) \\
=\frac{\partial^{2} u(t, \xi)}{\partial \xi^{2}} \\
+\int_{-\infty}^{t} a(t-s) u\left(s-\rho_{1}(t) \rho_{2}(\|u(t)\|), \xi\right) d s+B v(t) \\
\quad t \in[0, a], \quad \xi \in[0, \pi], \\
u(t, 0)=u(t, \pi)=0, \quad t \in[0, a], \\
u(t, \xi)=\phi(t, \xi) \quad \tau \leq 0,0 \leq \xi \leq \pi,
\end{gathered}
$$

where $\phi \in C_{0} \times L^{2}(h, X), 0<t_{1}<, \ldots, t_{n}<a$. For $y \in D(A)$, $y=\sum_{n=1}^{\infty}<y, \phi_{n}>\phi_{n}$, and $A y=-\sum_{n=1}^{\infty} n^{2}<y, n>\phi_{n}$, where $\phi_{n}(x)=\sqrt{2 / \pi} \sin n x, 0 \leq x \leq \pi, n=1,2,3, \ldots$ is the eigenfunction corresponding to the eigenvalue $\lambda_{n}=-n^{2}$ of the operator $A . \phi_{n}$ is an orthonormal base. $A$ will generate the operators $S(t), C(t)$ such that $S(t) y=\sum_{n=1}^{\infty}((\sin (n t)) / n)<$ $y, \phi_{n}>\phi_{n}, n=1,2, \ldots \forall y \in X$, and the operator $C(t) y=$ $\sum_{n=1}^{\infty} \cos (n t)<y, \phi_{n}>\phi_{n}, n=1,2, \ldots \forall y \in X$. Let the infinite dimensional control space be defined as $U=\{u: u=$ $\left.\sum_{n=2}^{\infty} u_{n} \phi_{n}, \quad \sum_{n=2}^{\infty} u_{n}^{2}<\infty\right\}$ with norm $\|u\|_{U}=\left(\sum_{n=2}^{\infty} u_{n}^{2}\right)^{1 / 2}$. Thus $U$ is a Hilbert space. By defining maps $\rho, G, F:[0, a] \times$ $\mathfrak{B} \rightarrow X$ by

$$
\rho(t, \psi):=\rho_{1}(t) \rho_{2}(\|\psi(0)\|)
$$

$$
\begin{gathered}
G(\psi)(\xi):=\int_{-\infty}^{0} \int_{0}^{\pi} b(s, v, \xi) \psi(s, v) d v d s, \\
F(\psi)(\xi):=\int_{-\infty}^{0} a(s) \psi(s, \xi) d s,
\end{gathered}
$$

the system (51) can be transformed into system (1). Assume that the functions $\rho_{i}: \mathbb{R} \rightarrow[0, \infty), a: \mathbb{R} \rightarrow \mathbb{R}$ are continuous and satisfy the following conditions.

(a) The functions $b(s, \eta, \xi), \partial b(s, \eta, \xi) / \partial \xi$ are measurable, $b(s, \eta, \pi)=b(s, \eta, 0)=0$ and

$$
\begin{aligned}
& L_{g} \\
& :=\max \left\{\left(\int_{0}^{\pi} \int_{-\infty}^{0} \int_{0}^{\pi} \frac{1}{h(s)}\left(\frac{\partial^{i} b(s, \eta, \xi)}{\partial \xi^{i}}\right)^{2} d \eta d s d \xi\right)^{1 / 2}:\right. \\
& \qquad i=0,1\}
\end{aligned}
$$

$<\infty$

such that $\|g\|_{\mathscr{L}(X)} \leq L_{g}$. 
(b) The function $F: \mathbb{R} \times \mathbb{R} \rightarrow \mathbb{R}$ is continuous and there is continuous function $L_{f}=\int_{-\infty}^{0}\left(a(s)^{2} / h(s)\right) d s<\infty$ and $\|F\|_{\mathscr{L}(X)} \leq L_{f}$.

(c) The functions $a_{i}^{j} \in C([0, \infty) ; \mathbb{R})$ and $L_{i}^{j}:=$ $\left(\int_{-\infty}^{0}\left(\left(a_{i}^{j}(s)\right)^{2} / h(s)\right) d s\right)^{1 / 2}<\infty$ for all $i=$ $1,2, \ldots, n j=1,2$.

Moreover $g(t, \cdot)$ is a bounded linear operator.

Here we examine the conditions (HR) for this control system. Then by using Theorem 18 we show its approximate controllability. Let $\widetilde{B}: U \rightarrow X: \widetilde{B} u=2 u_{2} \phi_{1}+\sum_{n=2}^{\infty} u_{n} \phi_{n}$ for $u=\sum_{n=2}^{\infty} u_{n} \phi_{n} \in U$. The bounded linear operator $B$ : $L_{2}([0, T] ; U) \rightarrow L_{2}([0, T] ; X)$ is defined by $(B u)(t)=\widetilde{B} u(t)$.

Let $\alpha \in N \subset L_{2}(0, T: X), N$ is the null space of $\Gamma$. Also $\alpha=\sum_{1}^{\infty} \alpha_{n}(s) \phi_{n}$. Therefore

$$
\int_{0}^{T} S(T-s) \alpha(s) d s=0 .
$$

This implies that

$$
\int_{0}^{T} \frac{\sin n(T-s)}{n} \alpha_{n}(s) d s=0, \quad n \in \mathcal{N} .
$$

The Hilbert space $L_{2}(0, T)$ can be written as

$$
L_{2}(0, T)=\operatorname{Sp}\{\sin s\}^{\perp}+\operatorname{Sp}\{\sin 4 s\}^{\perp} .
$$

Thus for $h_{1}, h_{2} \in L_{2}(0, T)$ there exists $\alpha_{1} \in\{\sin s\} \perp, \alpha_{2} \in$ $\{\sin 4 s\}^{\perp}$ such that $h_{1}-2 h_{2}=\alpha_{1}-2 \alpha_{2}$. So let $u_{2}=h_{2}-\alpha_{2}$. Then $h_{1}=\alpha_{1}+2 u_{2}, h_{2}=\alpha_{2}+u_{2}$ also let $u_{n}=h_{n}, n=3,4, \ldots$ and $\alpha_{n}=0, n=3,4, \ldots$. . Thus we see that hypothesis (HR) is satisfied as $U=\left\{u: u=\sum_{n=2}^{\infty} u_{n} \phi_{n}, \sum_{n=2}^{\infty} u_{n}^{2}<\infty\right\}$ and $\widetilde{B}: U \rightarrow X: \widetilde{B} u=2 u_{2} \phi_{1}+\sum_{n=2}^{\infty} u_{n} \phi_{n}$.

Hence by assumptions (a)-(c) and Theorem 18 it is ensured that the problem (51) is approximately controllable.

\section{Conflict of Interests}

The authors declare that there is no conflict of interests regarding the publication of this paper.

\section{Acknowledgments}

The authors would like to express sincere gratitude to the reviewer for his valuable suggestions. The first author would like to thank Ministry of Human Resource and Development with Grant no. MHR-02-23-200-429/304 for their funding.

\section{References}

[1] J. J. Nieto and D. O'Regan, "Variational approach to impulsive differential equations," Nonlinear Analysis: Real World Applications, vol. 10, no. 2, pp. 680-690, 2009.

[2] M. Benchohra, J. Henderson, and S. K. Ntouyas, Impulsive Differential Equations and Inclusions, Hindawi Publishing Corporation, New York, NY, USA, 2006.
[3] V. Lakshmikantham, D. D. Bainov, and P. S. Simeonov, Theory of Impulsive Differential Equations, World Scientific, Singapore, 1989.

[4] W. M. Haddad, V. Chellaboina, and S. G. Nersesov, Impulsive and Hybrid Dynamical Systems: Stability, Dissipativity, and Control, Princeton University Press, Princeton, NJ, USA, 2006.

[5] Y.-K. Chang, A. Anguraj, and M. M. Arjunan, "Existence results for impulsive neutral functional differential equations with infinite delay," Nonlinear Analysis: Hybrid Systems, vol. 2, no. 1, pp. 209-218, 2008.

[6] Y.-K. Chang and W.-T. Li, "Existence results for impulsive dynamic equations on time scales with nonlocal initial conditions," Mathematical and Computer Modelling, vol. 43, no. 3-4, pp. 377-384, 2006.

[7] B. Ahmad, "Instability of impulsive hybrid state dependent delay differential systems," Vietnam Journal of Mathematics, vol. 35, no. 3, pp. 285-298, 2007.

[8] A. Anguraj, M. M. Arjunan, and E. M. Hernández, "Existence results for an impulsive neutral functional differential equation with state-dependent delay," Applicable Analysis, vol. 86, no. 7, pp. 861-872, 2007.

[9] E. M. Hernández and M. A. McKibben, "On state-dependent delay partial neutral functional-differential equations," Applied Mathematics and Computation, vol. 186, no. 1, pp. 294-301, 2007.

[10] M. E. Hernández, M. Rabello, and H. R. Henríquez, "Existence of solutions for impulsive partial neutral functional differential equations," Journal of Mathematical Analysis and Applications, vol. 331, no. 2, pp. 1135-1158, 2007.

[11] E. Hernández, R. Sakthivel, and S. Tanaka Aki, "Existence results for impulsive evolution differential equations with statedependent delay," Electronic Journal of Differential Equations, vol. 28, pp. 1-11, 2008.

[12] W.-S. Li, Y.-K. Chang, and J. J. Nieto, "Solvability of impulsive neutral evolution differential inclusions with state-dependent delay," Mathematical and Computer Modelling, vol. 49, no. 9-10, pp. 1920-1927, 2009.

[13] M. Benchohra, G. M. N'Guérékata, and D. Seba, "Measure of noncompactness and nondensely defined semilinear functional differential equations with fractional order," Cubo, vol. 12, no. 3 , pp. 35-48, 2010.

[14] S. Ji and G. Li, "A unified approach to nonlocal impulsive differential equations with the measure of noncompactness," Advances in Difference Equations, vol. 2012, article 182, 2012.

[15] K. Balachandran and J. Y. Park, "Existence of solutions and controllability of nonlinear integrodifferential systems in Banach spaces," Mathematical Problems in Engineering, no. 1-2, pp. 6579, 2003.

[16] Z. Tai and X. Wang, "Controllability of fractional-order impulsive neutral functional infinite delay integrodifferential systems in Banach spaces," Applied Mathematics Letters, vol. 22, no. 11, pp. 1760-1765, 2009.

[17] R. Triggiani, "A note on the lack of exact controllability for mild solutions in Banach spaces," SIAM Journal on Control and Optimization, vol. 15, no. 3, pp. 407-411, 1977.

[18] J. P. Dauer and N. I. Mahmudov, "Approximate controllability of semilinear functional equations in Hilbert spaces," Journal of Mathematical Analysis and Applications, vol. 273, no. 2, pp. 310327, 2002.

[19] N. Sukavanam, "Approximate controllability of semilinear control of control system with growing nonlinearity," in Proceedings of the International Conference on Control Theory, pp. 353-357, Marcel Dekker, New York, NY, USA, 1993. 
[20] S. Das, D. N. Pandey, and N. Sukavanam, "Approximate controllability of a functional differential equation with deviated argument," Nonlinear Dynamics and Systems Theory, vol. 14, no. 3, pp. 265-277, 2014.

[21] D. Sanjukta, D. N. Pandey, and N. Sukavanam, "Exact controllability of an impulsive semilinear system with deviated argument in a banach space," Journal of Difference Equation, vol. 2014, Article ID 461086, 6 pages, 2014.

[22] S. Das, D. N. Pandey, and N. Sukavanam, "Approximate controllability of a second-order neutral differential equation with state dependent delay," Differential Equations and Dynamical Systems, 2014.

[23] J.-M. Jeong, J.-R. Kim, and H.-H. Roh, "Controllability for semilinear retarded control systems in Hilbert spaces," Journal of Dynamical and Control Systems, vol. 13, no. 4, pp. 577-591, 2007.

[24] E. Hernández and D. O'Regan, "On a new class of abstract impulsive differential equations," Proceedings of the American Mathematical Society, vol. 141, no. 5, pp. 1641-1649, 2013.

[25] E. Hernández, "Existence results for partial neutral functional integrodifferential equations with unbounded delay," Journal of Mathematical Analysis and Applications, vol. 292, no. 1, pp. 194210, 2004.

[26] E. Hernández and H. R. Henríquez, "Existence results for partial neutral functional differential equations with unbounded delay," Journal of Mathematical Analysis and Applications, vol. 221, no. 2, pp. 452-475, 1998.

[27] E. Hernández, M. Pierri, and G. Goncalves, "Existence results for an impulsive abstract partial differential equation with statedependent delay," Computers \& Mathematics with Applications, vol. 52, no. 3-4, pp. 411-420, 2006.

[28] H. O. Fattorini, Second Order Linear Differential Equations in Banach Spaces, North-Holland Math. Stud., 108, NorthHolland, Amsterdam, The Netherlands, 1985.

[29] J.-C. Chang and H. Liu, "Existence of solutions for a class of neutral partial differential equations with nonlocal conditions in the $\alpha$-norm," Nonlinear Analysis: Theory, Methods \& Applications, vol. 71, no. 9, pp. 3759-3768, 2009.

[30] J. K. Hale and J. Kato, "Phase space for retarded equations with infinite delay," Funkcialaj Ekvacioj, vol. 21, pp. 11-41, 1978.

[31] J. Banas and K. Goebel, Measure of Noncompactness in Banach Space, vol. 60 of Lecture Notes in Pure and Applied Mathematics, Marcel Dekker, New York, NY, USA, 1980.

[32] K. Balachandran and E. R. Anandhi, "Boundary controllability of integrodifferential systems in Banach spaces," Proceedings Mathematical Sciences, vol. 111, no. 1, pp. 127-135, 2001.

[33] Y.-K. Chang and J. J. Nieto, "Existence of solutions for impulsive neutral integro-differential inclusions with nonlocal initial conditions via fractional operators," Numerical Functional Analysis and Optimization, vol. 30, no. 3-4, pp. 227-244, 2009.

[34] Y. Hino, S. Murakami, and T. Naito, Functional Differential Equations with Infinite Delay, vol. 1473 of Lecture Notes in Mathematics, Springer, Berlin, Germany, 1991.

[35] C. C. Travis and G. F. Webb, "Cosine families and abstract nonlinear second order differential equations," Acta Mathematica Academiae Scientiarum Hungarica, vol. 32, no. 1-2, pp. 75-96, 1978.

[36] C. C. Travis and G. F. Webb, "Compactness, regularity, and uniform continuity properties of strongly continuous cosine families," Houston Journal of Mathematics, vol. 3, no. 4, pp. 555567, 1977.
[37] C. C. Travis and G. F. Webb, "Second order differential equations in Banach space," in Proceedings of an International Symposium on Nonlinear Equations in Abstract Spaces, pp. 331361, Academic Press, New York, NY, USA, 1978.

[38] K. Tsujioka, "Remarks on controllability of second order evolution equations in Hilbert spaces," SIAM Journal on Control and Optimization, vol. 8, pp. 90-99, 1970.

[39] K. Naito, "Controllability of semilinear control systems dominated by the linear part," SIAM Journal on Control and Optimization, vol. 25, no. 3, pp. 715-722, 1987. 


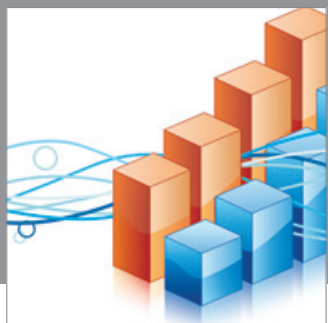

Advances in

Operations Research

mansans

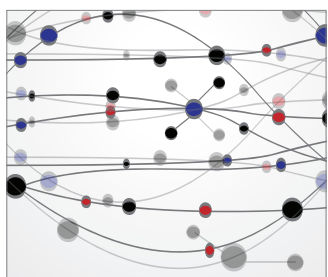

The Scientific World Journal
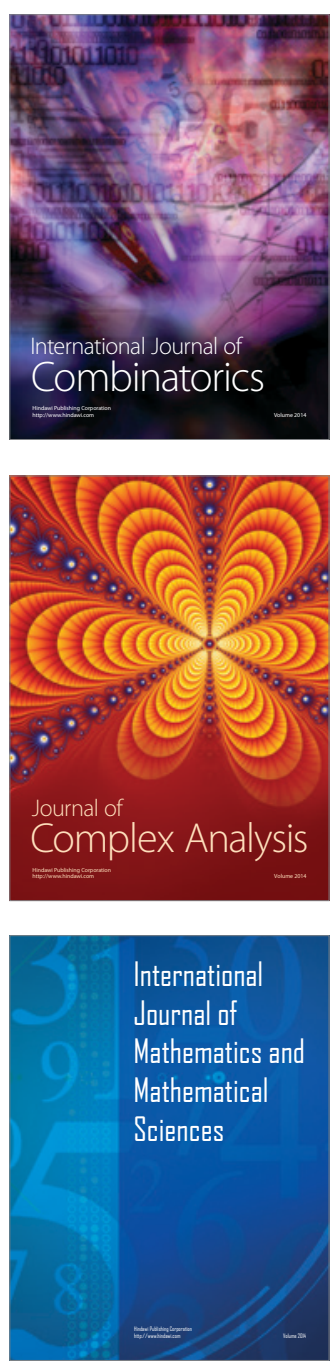
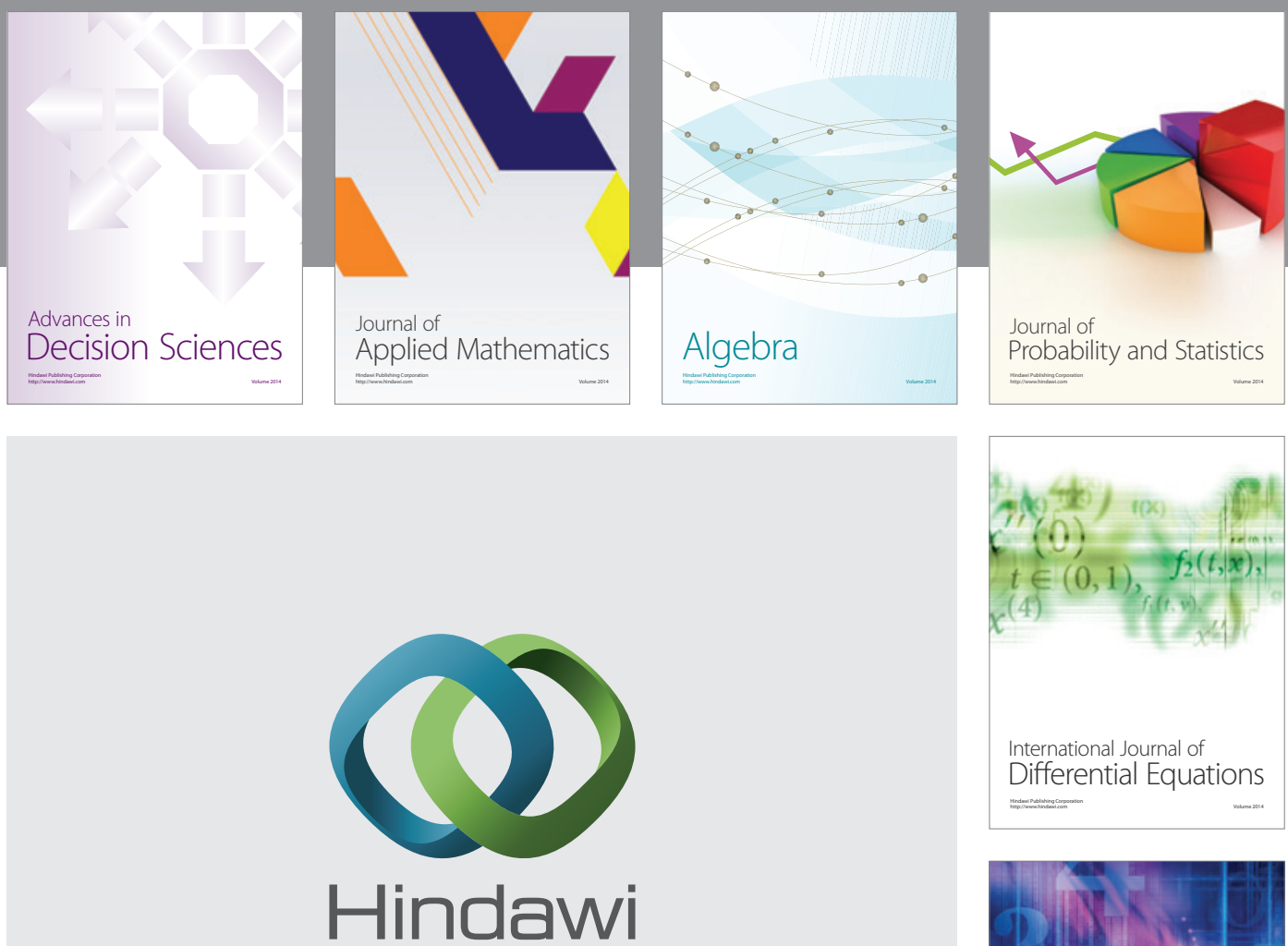

Submit your manuscripts at http://www.hindawi.com
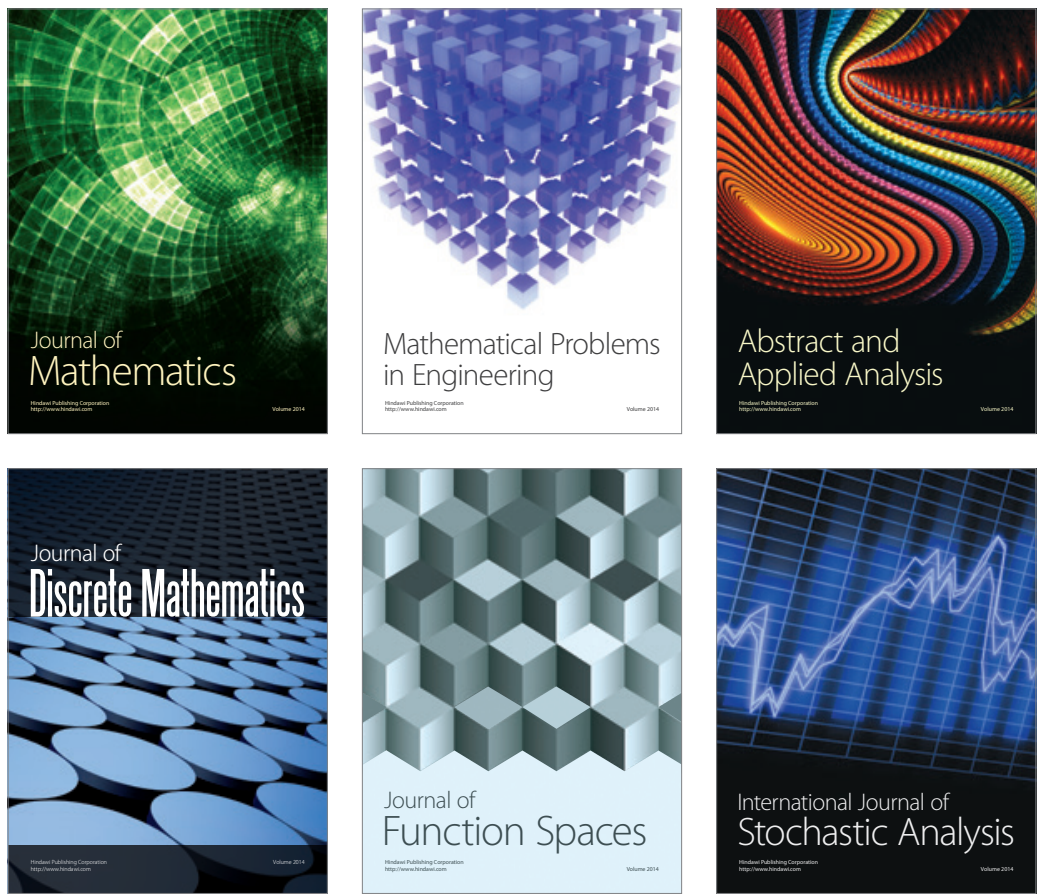

Journal of

Function Spaces

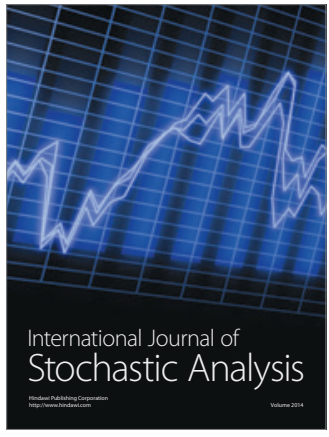

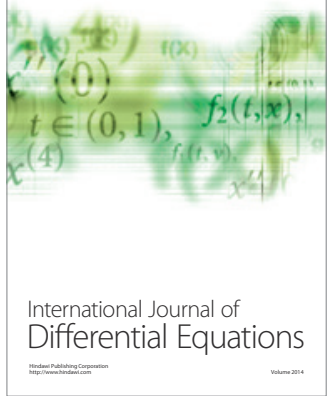
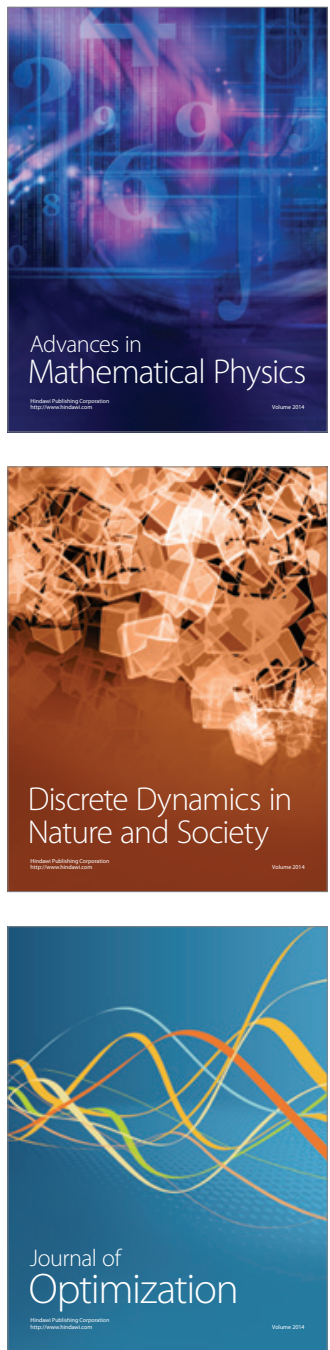